\title{
A Survey of Communication Network Paradigms for Substation Automation
}

\author{
Mahmood Qureshi, Ali Raza, Dileep Kumar, \\ Sang-Sig Kim, Un-Sig Song, \\ Min-Woo Park, and Hyuk-Soo Jang \\ Dept. of Computer Software, \\ Myongji University, Yongin, Korea \\ Email: mahmood, aliraza, dileep, \\ firewall007, unsigi, circle07, and jang-h@mju.ac.kr
}

\author{
Hyo-Sik Yang \\ Dept. of Computer Engineering \\ Sejong University, Seoul, Korea \\ Email: hsyang@sejong.ac.kr
}

\author{
Byung-Seok Park \\ Korea Electrical Power \\ Research Institute \\ (KEPRI) \\ Daejun, Korea \\ Email: blueon@kepri.re.kr
}

\begin{abstract}
In the realm of substation automation (SA), communication infrastructure plays a vital role in mediating between physical and virtual worlds of substation. Specification of data exchanges through standardized communication stacks is therefore an important issue for all substation equipment manufacturers seeking to provide vendor interoperability. Nowadays competitive electric utility marketplace, reliable and real-time information become the key factor for reliable delivery of power to the endusers, profitability of the electric utility and customer satisfaction. The operational and commercial demands of electric utilities require a high-performance data communication network that supports both existing functionalities and future operational requirements. As communication arena is changing day by day, the need for efficient and reliable communication infrastructure to address $\mathrm{SA}$ is evident. In this respect, a communication network constitutes the core of the $\mathrm{SA}$, thus the design of cost-effective and reliable network architecture is a crucial task. Most of the existing communication networks claim to address the need of communication architecture for SA but in some regard these claims just could not fulfill the constraints imposed by highly available environment for SA. This paper presents a survey and analysis of the current state-of-the-art communication infrastructure in the SA. As Ethernet technology becomes more reliable and also widely available with fiber optical communication so this paper also examines the key issues and requirements for Ethernet in the substation environment and also opens some research challenges.
\end{abstract}

\section{INTRODUCTION}

Substation Automation (SA) is a supervisory management and control system that lies in between transmission and distribution systems. The interests on SA have been increasing rapidly due to its numerous benefits to utilities. It has advanced further than a traditional Supervisory Control and Data Acquisition (SCADA) system providing additional capability and information that can be used to further improve operations, maintenance and efficiencies in substations. The most significant elements of a SA system include relays and/or Intelligent Electronic Devices (IEDs) that perform various control, monitoring and protection related operations.

The desire and the need of merging the communication capabilities of all relays and IEDs in a substation has thus been clearly recognized, which is capable of providing not only data gathering and setting capability but also remote control. Furthermore, multiple IEDs can share data or control commands at higher speeds to perform new distributed protection, and control functions. Interoperability needs to be achieved in a substation between protective relays from different manufacturers so that substation level interlocking, protection and control functions can be realized improving the efficiency of microprocessor based relay applications.

Recent developments in communication technologies have enabled reliable remote control systems which have the capability of monitoring the real time operating conditions and performance of electric systems. These communication technologies can be classified into four classes, i.e., power line communication, satellite communication, wireless communication, and optical fiber communication. Each communication technology has its own advantages and disadvantages that must be evaluated to determine the best communication infrastructure for SA.

Power line communication (PLC) utilizes the existing infrastructure of electricity for communication thereby offering a broad coverage as well as cost effective solution but on the other hand suffered by noise, capacity, signal distortion and security issues [1].

Whenever it comes to remote monitoring, satellite communication is opted as one of the available mean to achieve it [2]. Though satellite communication offers global coverage and rapid installation but performance stills the problem with unavoidable delay.

Optical fiber communication has taken the major share of communication due to its high capacity and immunity characteristics for EMI/RFI (Electro Magnetic Interference / Radio Frequency Interference). Though cost of deploying fiber optic communication is expensive but it is overcome by the advantages offered.

Wireless communication due to its rapid deployment and cost effective solution has dramatically change the trend of communication paradigm but still need to address the crucial issues of capacity, security, and coverage in order to make it viable.

In this paper, it is investigated that which communication architecture is feasible for SA. In this regard existing com- 
munication technologies such as power line communication, satellite communication, optical fiber communication, wireless communication, and sensor-net for SA are explored to search for the best available communication infrastructure.

The rest of this paper is organized as follows. Section II presents the current state-of-the-art communication infrastructure in the SA and also present analysis on them with respect to SA. Section III presents suggested communication architecture for SA and also proposes a future direction for research. Finally, Section IV summarizes the contribution of this paper.

\section{State of the ARt Communication Network}

In this section, a review of different communication technologies is presented with the special emphasis on their application for SA [3].

\section{A. Power Line Communication}

Power line communications (PLC) technology can use the household electrical power wiring as a transmission medium.. PLC uses the electrical wires of a home or building as the conduit to transmit data. Maximizing the use of existing infrastructures, PLC is a reliable and secure way to network. Setting Local Area Network (LAN) and connecting multiple computers to share Internet access, files, printers, or any other resources, has never been easier through PLC. PLC allows you to simply set up a network by plugging into any electrical outlet thereby providing a passage for instant connectivity. Commercially deployable, high speed and long distance PLC still requires further research efforts despite the fact that PLC might provide an alternative cost-effective solution to the last mile connectivity problem [1]. Following are the benefits of PLC when it comes for its application in SA:

1) Broad coverage: Broad Coverage is one of the major advantages of PLC. Power Lines are available almost everywhere in the world where there is a community of human beings. It also benefits the rural areas where communication infrastructure is not available. In addition, PLC boasts a superior distance of $300 \mathrm{~m}$ (without using repeaters) compared to $100 \mathrm{~m}$ for standard Fast-Ethernet and about $50 \mathrm{~m}$ for $802.11 \mathrm{~b}$ wireless connections.

2) High bandwidth: Current PLC technology has reached a maximum speed of $45 \mathrm{Mbps}$ [4]. This is divided into a maximum 27 Mbps for downstream speeds and $18 \mathrm{Mbps}$ for upstream speeds. PLC will have the highest access speeds, compared to ADSL clocking at $512 \mathrm{Kbps}$ and cable at 1.5 Mbps [5].

3) Cost-effective: Cost overhead on the user is negligible because it utilizes the existing wires to carry the communication signals. So this is among one of the cheapest methods available for remotely monitoring power usage.

4) Easy installation: As power lines are almost available everywhere in the world so it is very easy to install as compared to any other communication technology. It does not use extra wiring but existing wires of power lines thereby not posing installation problems in terms of resources and experts.
Despite these benefits of PLC there are some drawbacks too:

1) Lack of availability: Communication over the power lines may lose due to the open circuit of the installed devices [6]. This not only restricts the usefulness of PLC for applications especially involving switches, reclosers, and sectionalizes but also put a question mark on the highly available environment for SA [3].

2) Noise: Noise in data communication is one of the growing concerns when communication infrastructure is installed in the environment where electrical signals are generated and transmitted at a large scale. Thus it is not feasible to consider power lines as communication infrastructure for SAs since presence of high voltage devices in substation generate a noisy environment for communication [7]. These noise sources over the power lines can result in high bit error rates during communication which severely degrade the performance of PLC.

3) High Attenuation: The communication over power lines might be lost because of high signal attenuation and distortion or may degrade the reliability to take crucial steps in response to an event. Physical topology of the power network and load impedance fluctuation over the power lines are the sources for signal attenuation and distortion [8]. Power cables are not twisted and also not having proper shielding resulting fair amount of Electro Magnetic Interference (EMI) [9]. Thus by making use of radio receivers communication signals can be trapped easily or distorted severely by introducing EMI. Therefore, the proper encryption techniques must be used to prevent the interception of critical data by an unauthorized person.

\section{B. Satellite Communication}

Satellite communication can offer innovative solutions for remote control and monitoring of substations. It provides an extensive geographic coverage, and thus, can be a good alternative communication infrastructure for electric system automation in order to reach remote substations where other communication infrastructures such as telephone or cellular networks might not exist. In practical applications, Very Small Aperture Terminal (VSAT) satellite services are already available that are especially tailored for remote substation monitoring applications [10]. Furthermore, with the latest developments in electric system automation, satellite communication is not only used for remote control and monitoring of substations but also used for Global Positioning System (GPS) based time synchronization, which provides microsecond accuracy in time synchronization [11]. In addition, satellites can be used as a backup for the existing substations communication networks. In case of congestion or link failures in communication, critical data traffic can be routed through satellites [12]. In this section, both advantages and disadvantages of satellite communication technologies have been reviewed with respect to SA. Following are the advantages of using satellite communication for remote monitoring: 
1) Geographic coverage: Satellite communications have unique advantages over conventional long distance transmissions. This communication trend is capable of spanning long distance irrespective of the physical condition. Thus providing a cost-effective solution for the areas where communication infrastructure might not exist due to environmental issues.

2) Higher Bandwidth: Satellite communication has very large bandwidth capacity as it is widely used for the broadcasting of video streaming. Thus this higher bandwidth can be utilized for the transmission of mission critical data and messages within substations.

Followings are the disadvantages of using satellite communication as a communication technology:

1) Long delay: As for as the transmission characteristics for satellite communication are concerned, round-trip delay is the growing concern which is comparatively higher than the wired network. As substation messages are mission critical so in order to ensure efficient and reliable transmission of these message from one substation to other substation this round trip delay need to be minimized.

2) Satellite channel characteristics: Performance of satellite channels is greatly affected by the change in operating climate due to weather or by the effect of fading thereby degrading the efficiency of the whole satellite communication system [13].

\section{Optical Fiber Communication}

The Optical Fiber technology was first demonstrated in 1952 for internal medical. Later Optical fiber can be used as a medium for telecommunication and networking because of its flexibility can easily be bundled as cables. In case of SA, fiber optical communication is the most demanding and eyecatching communication infrastructure that gives very high speed.

Optical Fiber can easily carry high bandwidth data, making it possible to use with substations to share bandwidth capacity. This would also allow cost to be reduced because by sharing it with end users. Thereby offering high performance, reliable communication and greater coverage in the highly constrained environment of substations with respect to functional and performance parameters. Furthermore optical fiber is one the demanding communication medium for substations as characterized by high voltage operating environment because of its EMI/RFI immunity characteristics [6].

Now in the brief following are the advantages of using optical fiber communications as a communication medium.

1) Coverage: It is especially advantageous for longdistance communications, because light propagates through the fiber with little attenuation compared to electrical cables. For up to $2 \mathrm{Km}$ no need to amplify the signal or use repeater.

2) Light Weight: Optical fibers are light weight and small in size, which makes them ideal for applications where running copper wires would be impractical.
3) Security: Fiber Optical communications are very secure as compare with the other way of communications because it is very hard to tap into a fiber cable to read data signals [14].

4) Capacity: Extremely high bandwidth capacity of optical fiber communication can provide high performance communication for SA applications. Current optical fiber transmission systems provide transmission rates up to 10 Gbps using single wavelength transmission and 40 Gbps to $1600 \mathrm{Gbps}$ using wavelength division multiplexing (WDM). In addition, very low bit error rates (BER = $10^{-15}$ ) in fiber optic communication are observed. Due to high bandwidth capacity and low BER characteristics, optical fiber is used as the physical layer of Gigabit and 10 Gigabit Ethernet networks.

5) Immunity characteristics: Since Optical fibers are immune to energy radiation and possible interference from external sources [15]. Hence Optical fibers are more secure when it comes to signal trapping, crosstalk and EMI/RFI characteristics.

Now the only disadvantage of using optical fiber is cost. The cost of the optical fiber itself is still expensive to install for electric utilities. Its installation is also very expensive especially if it is used for remote controlling and monitoring of substations.

Even though the disadvantage is only the cost but dominant advantages will immediately affect the consumers' choice to install this technology for SA.

\section{Wireless Communication}

Wireless communication is the transfer of information over a distance without the use of electrical conductors or wires. Information is transferred in this manner over both short and long distances. Wireless technology carries the capabilities of wired networks to spaces where cable cannot be installed.

Several types of wireless technologies are present in the market for SA [6]. The main difference is their range. When compared to conventional wired communication networks, wireless communication technologies have potential benefits in controlling and monitoring remote substations, e.g., savings in cabling costs and rapid installation of the communication infrastructure. Security is one of the growing concerns in wireless communication since intelligent radio devices are available that can be used to trap wireless signals in the air thereby raising a great threat for secure wireless communication. Furthermore wireless communication system is greatly suffered by the limitations in bandwidth capacity, coverage and vulnerability to EMI.

In the brief the major benefits of wireless networks over wired networks are explored here:

1) Flexibility: The mobility of the devices within the environment is a simple matter to relocate a communicating device, and no additional cost of rewiring and excessive downtime is associated with such a move. It is also a simple matter to add in a communication device to 
the system or remove one from the system without any disruption to the remainder of the system.

2) Efficiency: In wireless technology, improved data communication lead to faster transfer of information and thereby increasing the wireless efficiency.

3) Cost: Initial outlay on setting up the cell sites, the cost of running and maintaining a radio based communication solution is minimal. Because wireless technology enables the user to communicate while on the move, so cabling cost is also eliminated.

4) Rapid installation: Wireless networks can be configured rapidly and installed as compared to wired networks. It can also be easier and cheaper to install. Thus communication can be initiated within the coverage by simply deploying short communication infrastructure.

Following are the drawbacks of using wireless network instead of wired network for communication:

1) Security: Wireless transmission is more vulnerable to attack by unauthorized users, poses serious security challenges, so particular attention has to be paid to security. Verification of communication entities must also be performed to ensure that only registered devices may communicate using the network, and that only registered devices may receive the data. Some form of encryption may be required for communication to avoid interception of data transmitted over the network by devices not taking part in the communication.

2) Interference problem: Interference problems seem often if others in the same building also use wireless technology or where other sources of radio signals are present. This could lead to poor communication or, in extreme cases, loss of wireless communication altogether. On the other hand wireless communication can also be lost due to congestion when too many wireless users interfere each other.

3) Limited coverage: In some buildings getting consistent coverage can be difficult, leading to "black spots" where no signal is available. For example, in structures built using steel reinforcing materials, one may find it difficult to pick up the radio frequencies used. Though public wireless networks such as cellular network, WIMAX and etc have greater coverage as compared with private wireless networks, the coverage of IEEE $802.11 \mathrm{~b}$ is approximately $100 \mathrm{~m}$ [16] but as in case of remote substation in less densely populated area wireless communication services may not have been deployed [14].

4) Capacity: Wireless communication technologies provide typically lower QoS (Quality of Service) compared to wired communication networks. It is also potential for radio interference due to weather, a limited bandwidth capacity is supported and high bit error rates are observed in communication. The application average data rate per end user is lower than the total bandwidth capacity, e.g., maximum data rate of IEEE 802.11 b is $11 \mathrm{Mbps}$ while the average application data rate is approximately $6 \mathrm{Mbps}$ [16]. Similarly, the 54Mbps maximum of $802.11 \mathrm{a}$ and $802.11 \mathrm{~g}$ works out nearer $20 \mathrm{Mbps}$ in real-life applications, in both cases the wireless networking is operating as slow as $10 \mathrm{Mbps}$ even under good conditions [17].

\section{E. Wireless Sensor Network Communication}

In the realm of remote monitoring and surveillance, wireless sensor networks play a vital role in mediating between physical world and computational world. The rapid development in communication electronics paves the way for the large scale deployment of sensor networks in every walk of life. Wireless sensor networks are driven by low cost, low power and multifunctional sensor nodes over a limited distance [15]. These sensor nodes collect data for monitoring and surveillance purposes such as weather monitoring, health care, traffic analysis, irrigation and etc. Efficiency, coverage, scalability, and fault-tolerance are one of the prominent characteristics of wireless senor network those can have significant impact on the automation process of SA.

The advantages of using sensor networks for telecommunication in SA are summarized below:

1) Surveillance in harsh conditions: Reliability, easy deployment, and self-configuring of sensor nodes in extreme environment make wireless sensor network extremely popular in communication arena. Thus, WSNs can operate even in harsh environments and eliminate the cabling requirement in substation systems where high voltage lines and devices are already deployed.

2) Coverage: Wireless sensor networks can be distributed over a large spatial region with limited deployment resources. As a single sensor node can monitor just a limited distance as per specification but the collaborative approach of sensor network can give a wider coverage. Thus, WSNs can be useful to monitor remote substations or high-voltage lines with broader coverage.

3) Improved accuracy: Accuracy in wireless sensor network can be guaranteed by making use of collaborative approach to monitor the target area with their physical limitation as compared to traditional monitoring devices [15].

4) Intelligent sensing: By using efficient data processing architecture, raw data at sensor node can further be processed according to given requirements prior to the transmission to remote gateway thereby making efficient use of resources such as reducing overhead for communication.

5) Low cost: Nowadays pervasive use of wireless sensor networks for monitoring is because of their low cost and for ease of their deployment.

6) Easy Maintenance: Because of dynamic behavior and self-configuration, wireless sensor networks can be deployed rapidly and maintained at the expense of very low resources irrespective of affecting the general objective of monitoring system as a whole.

Disadvantages of using WSN for surveillance are: 
1) Limited resources: Due to limited resources such as energy, memory, and processing available on the sensor node, it is a challenging job to optimize the network architecture for the monitoring applications to make best use of limited resources.

2) QoS concern: As QoS for WSNs deals with how much accuracy is assured by wireless sensor networks for the monitoring environment. As sensor data is time critical, if due to network latency or unavailability of a sensor node, sensor data is not reached at gateway, then monitoring accuracy can be compromised and decision is made on correlated data.

3) Dynamic topologies: Availability of sensor node becomes one of the rising concerns when wireless sensor network is deployed for any application. Because of unavailability of sensor node, the communication architecture for sensor network remains no more fixed rather keep on changing as sensor node goes down because of any failure, limited power, or some malicious activity. Hence, efficient communication protocols need to be evolved to deal with the network dynamics [18].

\section{Suggested Communication Paradigm}

Communication infrastructures obviously have the potential to virtualize the physical world of SA. However there are many challenges that need to be overcome for the complete realization of SA. This section explores the viable communication paradigm for SA and the most important challenges in this regard. The key assumptions, analyzed while exploring the existing communication networks in Section II, for substation are capacity, performance, coverage, security, reliability, accuracy, and availability that need to be addressed by the suggested communication paradigm for SA. As IEC 61850 has standardized the automation process of SA, so according to IEC 61850 Ethernet packet is the choice to transmit the substation message within the substation because of its performance and also most of the proprietary communication solutions are available with the Ethernet support [19]. Recently Ethernet technology has changed the trend of communication network because of its availability, efficiency, and cost effectiveness. Now Ethernet switches are being deployed for long haul communication access networks. As the local substation messages are inherently available in Ethernet format so these messages can be transmitted to other substation without incurring the cost of protocol conversion at access networks thereby insuring efficiency. This is one of the primal advantages of using Ethernet technology in substation. On the other hand optical fiber communication systems have dramatically taken the attention as a desired mode of communication due to its high capacity, efficiency, and security. Nowadays different projects are being run to make use of optical fiber at large scale such as FTTH (Fiber To The Home), FFTO (Fiber To The Office), and FTTC (Fiber To The Curb). Now what we suggest as the desired communication paradigm for SA is the combination of Ethernet as a communication protocol, to efficiently transmit the substation messages, and fiber optical communication system as a communication medium to ensure efficiency, coverage, and security. EPON is the desired communication paradigm that meets SA needs as it uses Ethernet as communication protocol and fiber optical communication system as communication medium. EPON also known as Ethernet in the First Mile (EFM) is a collection of protocols specified in IEEE 802.3. [20]. EPON is an emerging access network technology that provides a low-cost method of deploying optical access lines. Build on the International Telecommunications Union (ITU) standard G.983 for asynchronous transfer mode PONs (APON). The IEEE 802.3 standard defines two basic configurations for an Ethernet network. In one case it can be deployed over shared medium using carrier sense multiple access with collision detection (CSMA/CD) protocol [20]. In another case stations may be connected through a switch using full-duplex links. Properties of an EPON are such that it cannot be considered either shared medium or a point to point network; rather it is a combination of both [21]. As per specification of IEC61850, substation can be logically divided into three functional levels, i.e., Station level, process level and bus level. Station level is responsible for controlling and protection of entire substation. Devices such as HMI, engineering, control center, remote control center, and etc, are station level devices. Bay level devices are responsible for control, protection, and monitoring units related to different subparts of substation. Process level devices such as I/Os, intelligent sensors, and actuators are responsible for providing interface to the primary equipments of substation [19]. Accordingly, our suggested communication paradigm is modeled across three logical levels of substation as specified by IEC61850. In Fig. 1, lowest level communication model for SA is shown, i.e., communication model for bay level and process level. Bay level devices such as IEDs are interfaced with process level devices such as CT/VTs (Current Transformer / Voltage Transformers), CB (Circuit Breaker) and switch gear using Gigabit Ethernet switch.

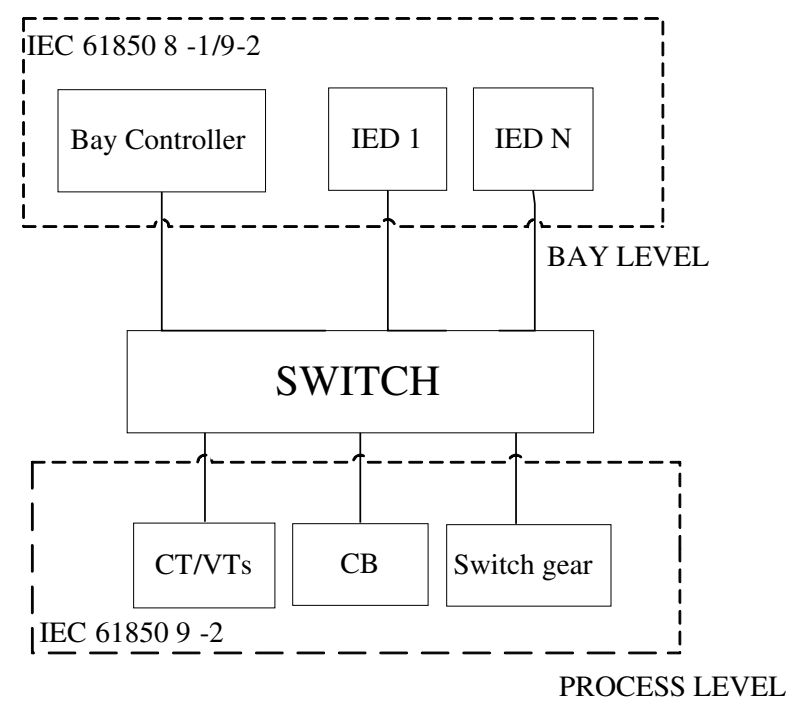

Fig. 1. Ethernet based SAS architecture. 
In Fig. 2, Communication model for station level and bay level is shown for the entire substation. Gigabit Ethernet is deployed between station level devices and bays. Different bay level switches are connected to station level devices via primary switch and if primary switch goes down in case of failure then these bays are connected through fault tolerant switch to station level devices. Primary switch and fault tolerant switch is in turn connect to ONU (Optical Network Unit) for access network via router and firewall, if there is any problem at access network then wireless communication can be used to back up access network.

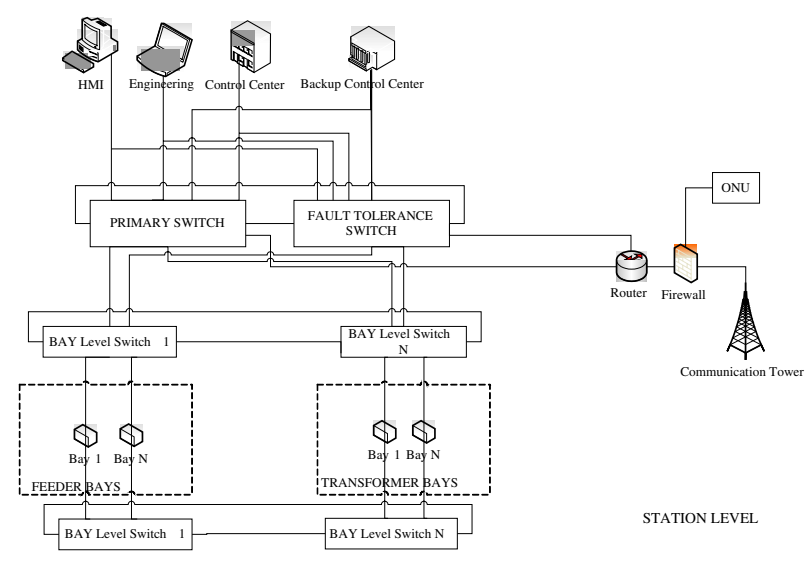

Fig. 2. Giga-bit optical Ethernet based SAS rchitecture.

EPON based SA is illustrated in Fig 3. ONU is the interface between the real substation and the PON; it receives traffic in optical format and converts it to the real substation messages, i.e., in Ethernet format. ONU also provides Layer-2 and Layer-3 switching functionality thereby allowing internal routing of traffic at the ONU. OLT (Optical Line Terminal) device resides in the central office or cable company head end. It generates or passes traffic on TDM based DWDM signals via fiber to an ONU [19].

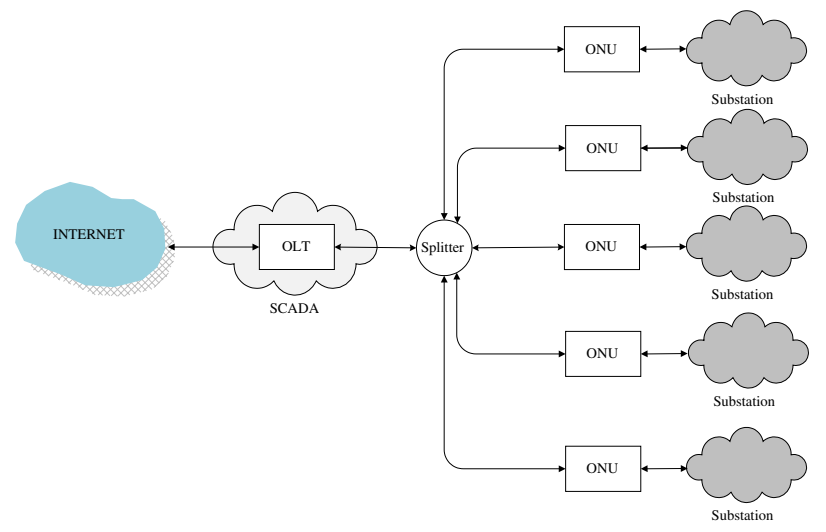

\section{CONCLUSION}

In this paper, an overview of the current state-of-the-art in communication paradigm for SA is presented. Existing communication infrastructures have some shortcomings in order to address the viable communication solution for the highly critical environment of SA. As Ethernet technology is becoming extremely popular because of cost and high availability and on the other hand fiber optic is emerging as the technically most attractive communication medium. We proposed EPON based architecture as the desired communication paradigm for the SA which is shipped with the two most demanding communication trends, i.e., Ethernet and fiber optic in a single package.

\section{REFERENCES}

[1] I. H. Cavdar, "A solution to remote detection of illegal electricity usage via power line communications," IEEE Trans. on Power Delivery, vol. 19, no. 4, pp. 1663-1667, Oct. 2004.

[2] B. G. Evans, Satellite Communication Systems, Inst. of Engineering and Technology, 2004.

[3] V. C. Gungor and F. C. Lambert, "A survey on communication networks for electric system automation," Computer Networks, vol. 50, no. 7, pp. 877-897, May 2006.

[4] Y. Abe et al., "Development of high speed power line communication modem," Tech. Rep., SEI Tech. Review, June 2004.

[5] O. G. Lee, "Focus: Pwer grid access [online]," Network Magazine, Aug. 2002.

[6] F. Goodman et al., "Technical and system requirements for advanced distribution automation,” Tech. Rep., Electric Power Research Inst., June 2004.

[7] N. Pavlidou, A. J. H. Vinck, J. Yazdani, and B. Honary, "Power line communications: State of the art and future trends," IEEE Comm. Mag., vol. 41, no. 4, pp. 34-40, Apr. 2003.

[8] S. Galli, A. Scaglione, and K. Dosterl, "Broadband is power: Internet access through the power line network," IEEE Comm. Mag., vol. 41, no. 5, pp. 82-83, May 2003.

[9] W. Liu, H. Widmer, and P. Raffin, "Broadband PLC access systems and field deployment in european power line networks," IEEE Comm. Mag., vol. 41, no. 5, pp. 114-118, May 2003.

[10] A. Tisot, "Rio grande electric monitors remote energy assets via satellite," Utility Automation \& Engineering T\&D Mag., July 2004.

[11] G. E. Ziegler, "Protection and substation automation," Electra, vol. 206, pp. 14-23, Feb. 2003.

[12] E. Ekici, I. F. Akyildiz, and M. D. Bender, "A multicast routing algorithm for LEO satellite IP networks," IEEE/ACM Trans. on Networking, vol. 10, no. 2, pp. 183-192, Apr. 2002.

[13] Y. Hu and V. O. K. Li, "Satellite-based internet: A tutorial," IEEE Comm. Mag., vol. 39, no. 3, pp. 154-162, Mar. 2001.

[14] B. Mukherjee, Optical Communication Networks, McGrow-Hill, 1997.

[15] I. F. Akyildiz, W. Su, Y. Sankarasubramaniam, and E. Cayirci, "Wireless sensor networks: A survey," Computer Networks, vol. 38, pp. 393-422, Apr. 2002

[16] J. Jun, P. Peddabachagari, and M. Sichitiu, "Theoretical maximum throughput of IEEE 802.11 and its applications," in IEEE Int. Symposium on Network Computing and Applications, Apr. 2003, pp. 249-256.

[17] J. Regis, Broadband Telecommunications Handbook, McGraw-Hill, 2002.

[18] O. B. Akan M. C. Vuran, V. C. Gungor, "On the interdependence of congestion and contention in wireless sensor networks," in ICST Conference, July 2005.

[19] IEC 61850, "Communication networks and system in substations," 2002-2005, Available at http: //www. uac.ch.

[20] M. McGarry, M. Maier, and M. Reisslein, "WDM ethernet passive optical networks (EPONs)," IEEE Comm. Mag., vol. 5, no. 9, pp. 1825, Feb. 2006

[21] G. Kramer and G. Pesavento, "Ethernet passive optical network EPON: Building a next generation optical access network," IEEE Comm. Mag., vol. 40, no. 2, pp. 66-73, Feb. 2002.

Fig. 3. EPON based SAS architecture. 